\title{
A GAP TAUBERIAN THEOREM FOR GENERALISED ABSOLUTE ABEL SUMMABILITY
}

\author{
K. SARVOTHAMAN
}

ABSTRACT. A gap Tauberian theorem for generalised absolute Abel summability $\left|A_{\alpha}\right|$ is proved using Mel'nik's theorem on convolution transforms.

1. Introduction. The well-known gap Tauberian theorem for Abel summability $\left(A_{0}\right) \equiv(A)$ is a special case of the high indices theorem of Hardy and Littlewood [2, Theorem 114]. The gap Tauberian theorem for $\left(A_{\alpha}\right)$ summability has been proved by Krishnan [3]. Zygmund [5] has proved that $\left|A, \lambda_{n}\right|$ summability implies absolute convergence when $\left(\lambda_{n}\right)$ satisfies the high indices condition $\lambda_{n+1} / \lambda_{n} \geqslant c>1$, and Mel'nik [4] had deduced the same result as a corollary of his general theorem, which is stated here as Lemma 1. The gap Tauberian theorem for absolute Abel summability $\left|A_{0}\right| \equiv|A|$ is a special case of Zygmund's result when we take $\left(\lambda_{n}\right)$ as a sequence of integers. The purpose of this note is to show that the gap Tauberian theorem for absolute $A_{\alpha}$ summability $\left|A_{\alpha}\right|$ can be deduced from Mel'nik's theorem.

2. Definitions and notations. Let $\alpha>-1$. For a given series $\sum_{n=0}^{\infty} a_{n}$, write

$$
\begin{aligned}
A_{n} & =\sum_{r=0}^{n} a_{r} \quad(n \geqslant 0), \\
\bar{A}(y) & =\sum_{n=y} a_{n}, \\
a(x) & =\sum_{n=0}^{\infty} a_{n}\left(\begin{array}{c}
n+\alpha \\
\alpha
\end{array}\right) x^{n} \quad(0<x<1), \\
A(x) & =\sum_{n=0}^{\infty} A_{n}\left(\begin{array}{c}
n+\alpha \\
\alpha
\end{array}\right) x^{n} \quad(0<x<1), \\
f(x) & =(1-x)^{\alpha+1} A(x) \quad(0<x<1) .
\end{aligned}
$$

We assume that the series defining $a(x)$ and $A(x)$ converge for $0<x<1 . \sum a_{n}$ is summable $\left(A_{\alpha}\right)$ to $A$ if

$$
f(x)=(1-x)^{\alpha+1} A(x) \rightarrow A \text { as } x \rightarrow 1-,
$$

Received by the editors September 20, 1982. Presented to the annual conference of the Indian Mathematical Society, December 27, 1981.

1980 Mathematics Subject Classification. Primary 40F05; Secondary 40E15, 40G10.

Kel words and phrases. Absolute summability, gap condition, integral transforms.

¿1983 American Mathematical Society $0002-9939 / 83 \$ 1.00+\$ .25$ per page 
and absolutely $A_{\alpha}$ summable to $A\left(\left|A_{\alpha}\right|\right.$ summable to $\left.A\right)$ if

$$
\int_{0}^{1}\left|\frac{d}{d x} f(x)\right| d x<\infty
$$

and

$$
f(x)=(1-x)^{\alpha+1} A(x) \rightarrow A \quad \text { as } x \rightarrow 1-.
$$

Abel summability and absolute Abel summability are, respectively, $A_{0}$ and $\left|A_{0}\right|$ summability methods.

We say, following Mel'nik, that the function $s(v)$ belongs to the class $\left|T_{0}\right|$ if (i) $s(v)$ is of bounded variation in every finite interval; (ii) there exist constants $\mu, \delta$ and a function $\theta(v)$, depending only on $v$, such that $0<\mu, \delta \leqslant 1$, and the inequality

$$
\operatorname{Re}\left\{e^{i \theta} d s(u)\right\} \geqslant \mu|d s(u)|
$$

is satisfied for all $u$ in $[v-\delta, v+\delta]$.

3. Theorem and lemmas. The main result is

THEOREM. If $\sum a_{n}$ is summable $\left|A_{\alpha}\right|$ and satisfies a gap condition

(G) $a_{n}=0$ for $n \neq n_{k}$, where $\left(n_{k}\right)$ is a sequence of positive integers such that $n_{0}>0, n_{k+1} / n_{k} \geqslant c>1$ for all $k=0,1,2, \ldots$,

then $\sum a_{n}$ converges absolutely.

We first prove some preliminary lemmas.

LEMMA 1. Let the kernel $k(u)$ of the integral transform

$$
g(v)=\int_{-\infty}^{\infty} k(v-u) d s(u)
$$

be Borel measurable,

$$
\sum_{n=-\infty}^{\infty} \sup _{n \leqslant u<n+1}|k(u)|<\infty,
$$

and its Fourier transform

$$
K(t)=\int_{-\infty}^{\infty} e^{-i u t} k(u) d u \neq 0
$$

for $t \in E=(-\infty, \infty)$. Also assume $s(u)$ belongs to the class $\left|T_{0}\right|$ and

$$
\int_{v}^{v+1}|d s(u)| \leqslant M \text { for } v \in E .
$$

Then we can find a constant $C$ depending only on $\delta, \mu$ and $k(u)$ such that

$$
\int_{-\infty}^{\infty}|d s(v)| \leqslant C \int_{-\infty}^{\infty}|g(v)| d v .
$$

This is contained in Mel'nik's theorem. [4, Theorem 1].

LEMMA 2. If $\sum a_{n}$ is summable $\left|A_{\alpha}\right|$ and satisfies the gap condition $(\mathrm{G})$, then

$$
\int_{0}^{1}\left|f^{\prime}(x)\right| d x=\int_{-\infty}^{\infty}\left|g_{1}(v)\right| d v
$$


where

$$
\begin{gathered}
g_{1}(v)=\int_{-\infty}^{\infty} k_{1}(v, u) d s(u), \\
s(u)=\bar{A}\left(e^{u}\right), \\
\text { (8) } k_{1}(v, u)= \begin{cases}\frac{\left\{1-\exp \left(e^{-v}\right)\right\}^{\alpha} e^{-v} \exp \left\{-e^{u-v}\right\} \Gamma\left(\alpha+e^{u}+1\right)}{\Gamma(1+\alpha) \Gamma\left(e^{u}\right)} & \text { for } u \geqslant 0, \\
k(v-u) & \text { for } u<0,\end{cases}
\end{gathered}
$$

and

$$
k(t)=\exp \left\{-(\alpha+1) t-e^{-t}\right\} / \Gamma(\alpha+1) .
$$

Proof. First we find an expression for $f^{\prime}(x)$ in terms of $a_{n}$.

$$
\begin{aligned}
& f^{\prime}(x)=-(\alpha+1)(1-x)^{\alpha} \sum_{n=0}^{\infty}\left(\begin{array}{c}
n+\alpha \\
\alpha
\end{array}\right) A_{n} x^{n}+(1-x)^{\alpha+1} \sum_{n=0}^{\infty}\left(\begin{array}{c}
n+\alpha \\
\alpha
\end{array}\right) A_{n} n x^{n-1} \\
& =(1-x)^{\alpha}(\alpha+1)\left[-\sum_{n=0}^{\infty}\left(\begin{array}{c}
n+\alpha \\
\alpha
\end{array}\right) A_{n} x^{n}+(1-x) \sum_{n=1}^{\infty}\left(\begin{array}{c}
n+\alpha \\
n-1
\end{array}\right) A_{n} x^{n-1}\right] \\
& =(1-x)^{\alpha}(\alpha+1)\left[-\sum_{n=0}^{\infty}\left(\begin{array}{c}
n+\alpha \\
\alpha
\end{array}\right) A_{n} x^{n}\right. \\
& \left.+\sum_{n=1}^{\infty}\left(\begin{array}{l}
n+\alpha \\
n-1
\end{array}\right) A_{n} x^{n-1}-\sum_{n=1}^{\infty}\left(\begin{array}{c}
n+\alpha \\
n-1
\end{array}\right) A_{n} x^{n}\right] \\
& =(1-x)^{\alpha}(\alpha+1) \sum_{n=0}^{\infty}\left[-A_{n}\left\{\left(\begin{array}{c}
n+\alpha \\
\alpha
\end{array}\right)+\left(\begin{array}{c}
n+\alpha \\
n-1
\end{array}\right)\right\}+A_{n+1}\left(\begin{array}{c}
n+1+\alpha \\
n
\end{array}\right)\right] x^{n} \\
& =(1-x)^{\alpha}(\alpha+1) \sum_{n=0}^{\infty}\left(\begin{array}{c}
n+1+\alpha \\
n
\end{array}\right) a_{n+1} x^{n} \\
& =(1-x)^{\alpha}(\alpha+1) \sum_{n=1}^{\infty}\left(\begin{array}{l}
n+\alpha \\
n-1
\end{array}\right) a_{n} x^{n-1} \\
& =(1-x)^{\alpha}(\alpha+1) \int_{1}^{\infty} \frac{\Gamma(y+\alpha+1)}{\Gamma(y) \Gamma(\alpha+2)} x^{y-1} d \bar{A}(y) \\
& =(1-x)^{\alpha} \int_{1}^{x} \frac{\Gamma(y+\alpha+1)}{\Gamma(y) \Gamma(\alpha+1)} x^{y-1} d \bar{A}(y) \text {. }
\end{aligned}
$$

Substituting $x=\exp \left\{-e^{-v}\right\}, y=e^{u}$, we obtain

$$
f^{\prime}(x)=F(v)=\int_{0}^{x} \frac{\left\{1-\exp \left(-e^{-v}\right)\right\}^{\alpha} \Gamma\left(\alpha+e^{u}+1\right)}{\Gamma(1+\alpha) \Gamma\left(e^{u}\right)} \frac{\exp \left(-e^{u-v}\right)}{\exp \left(-e^{-v}\right)} d \bar{A}\left(e^{u}\right) .
$$

Hence,

$$
\int_{0}^{1}\left|f^{\prime}(x)\right| d x=\int_{-\infty}^{\infty}|F(v)| \frac{d x}{d v} d v=\int_{-\infty}^{\infty}\left|g_{1}(v)\right| d v,
$$


where

$$
\begin{aligned}
g_{1}(v)= & F(v) \frac{d x}{d v} \\
= & \int_{0}^{\infty} \frac{\left\{1-\exp \left(-e^{-v}\right)\right\}^{\alpha} \Gamma\left(\alpha+e^{u}+1\right)}{\Gamma(1+\alpha) \Gamma\left(e^{u}\right)} \frac{\exp \left(-e^{u-v}\right)}{\exp \left(-e^{-v}\right)} \exp \left(-e^{-v}\right) e^{-v} d \bar{A}\left(e^{u}\right) \\
= & \int_{0}^{\infty} \frac{\left\{1-\exp \left(-e^{-v}\right)\right\}^{\alpha} e^{-v}}{\Gamma(1+\alpha) \Gamma\left(e^{u}\right)} \exp \left\{-e^{u-v}\right\} \Gamma\left(\alpha+e^{u}+1\right) d \bar{A}\left(e^{u}\right) \\
& +\int_{-\infty}^{0} k(v-u) d \bar{A}\left(e^{u}\right),
\end{aligned}
$$

because $a_{0}=0$ under the assumption of the gap condition $(\mathrm{G})$, and hence $\bar{A}\left(e^{u}\right)=0$ for $u<0$. Here $k(t)$ is given by (10). Hence,

$$
\int_{0}^{1}\left|f^{\prime}(x)\right| d x=\int_{-\infty}^{\infty} k_{1}(v, u) d s(u),
$$

where $s(u)$ and $k_{1}(v, u)$ are given by (8) and (9).

Mel'nik [4, p. 834] has observed that his theorem can be applied to functions $g_{1}(v)$ which are expressible as

$$
g_{1}(v)=\int_{-\infty}^{\infty} k_{1}(v, u) d s(u)
$$

where $k_{1}(v, u)$ is not of the canonical form $k(v-u)$ as in (1) but can be "approximated" to a canonical form in a certain sense. The context of Lemma 3 below is that the kernel $k_{1}(v, u)$ appearing in (7) can be approximated in this sense. In the proof of the Theorem in $\$ 4$ we incorporate the details as to how this approximation is useful.

LEMMA 3. If $k(t)$ and $k_{1}(v, u)$ are the kernels of Lemma 2 given by (10) and (9), respectively, and $-1<\alpha<0$, then

$$
\sum_{n=-\infty}^{\infty} \max _{n \leqslant u<n+1} \int_{-\infty}^{\infty}\left|k_{1}(v, u)-k(v-u)\right| d v=L<\infty .
$$

Proof. For $u<0, k_{1}(v, u)=k(v-u)$ and therefore it suffices to prove

$$
\sum_{n=0}^{\infty} \max _{n \leqslant u<n+1} \int_{-\infty}^{\infty}\left|k_{1}(v, u)-k(v-u)\right| d v=L<\infty .
$$

Let $u \geqslant 0$. Then

$$
\begin{aligned}
& k_{1}(v, u)-k(v-u)= \frac{\left\{1-\exp \left(-e^{-v}\right)\right\}^{\alpha} e^{-v} \exp \left\{-e^{u-v}\right\} \Gamma\left(\alpha+e^{u}+1\right)}{\Gamma(1+\alpha) \Gamma\left(e^{u}\right)} \\
&-\frac{\exp \left\{(\alpha+1)(u-v)-e^{u-v}\right\}}{\Gamma(1+\alpha)} \\
&= \frac{(1-x)^{\alpha}\left(\log x^{-1}\right) x^{y} \Gamma(\alpha+y+1)}{\Gamma(1+\alpha) \Gamma(y)}-\frac{y^{\alpha+1}\left(\log x^{-1}\right)^{\alpha+1} x^{y}}{\Gamma(1+\alpha)} \\
&(12) \quad\left(\text { where } y=e^{u}, x=\exp \left\{-e^{-v}\right\}, y \geqslant 1\right) \\
&=x\left(\log \frac{1}{x}\right)\left[\frac{(1-x)^{\alpha} \Gamma(\alpha+y+1)}{\Gamma(y) \Gamma(1+\alpha)}-\frac{\left(\log x^{-1}\right)^{\alpha} y^{\alpha+1}}{\Gamma(1+\alpha)}\right] .
\end{aligned}
$$


Now

$$
\Gamma(\alpha+y+1) / \Gamma(y)=y^{\alpha+1}+O\left(y^{\alpha}\right) \text { uniformly in } y \geqslant 1 .
$$

Also

$$
\log x^{-1}=(1-x)+O(1-x)^{2} \text { uniformly in } \delta<x<1
$$

and, since $\alpha<0$, it follows that

$$
\left(\log x^{-1}\right)^{\alpha}=(1-x)^{\alpha}+O(1-x)^{\alpha+1}
$$

uniformly in $0<x<1$. It follows that, uniformly in $y \geqslant 1,0<x<1$, (12) is

$$
x^{y} \log x^{-1}\left\{O\left(y^{\alpha}(1-x)^{\alpha}\right)+O\left(y^{\alpha+1}(1-x)^{\alpha+1}\right)\right\} .
$$

Hence, uniformly in $y \geqslant 1$, we have

$$
\begin{aligned}
\int_{-\infty}^{\infty}\left|k_{1}(v, u)-k(v-u)\right| d v= & O\left\{y^{\alpha} \int_{0}^{1} x^{y-1}(1-x)^{\alpha} d x\right\} \\
& +O\left\{y^{\alpha+1} \int_{0}^{1} x^{y-1}(1-x)^{\alpha+1} d x\right\} \\
= & O(1 / y)=O\left(e^{-u}\right) .
\end{aligned}
$$

The lemma follows.

4. Proof of the Theorem. By Theorems 2 and 5 of [1], summability $\left|A_{\lambda}\right|$ implies $\left|A_{\mu}\right|$ for $\lambda>\mu>-1$. Hence it suffices to prove the theorem for $-1<\alpha<0$. Define

$$
g(v)=\int_{-\infty}^{\infty} k(v-u) d s(u)
$$

and apply Lemma 1 to $g(v)$. It has the canonical form (1). (2) can be easily verified. The Fourier transform of $k(x)$ is

$$
K(t)=\Gamma(\alpha+1+i t) / \Gamma(\alpha+1) \neq 0
$$

for any $t$, hence (3) is satisfied. If (G) is satisfied, and if $\delta>0$ is sufficiently small, it follows that for any $v$ the interval $[v-\delta, v+\delta]$ contains not more than one point at which the function $s(u)=\bar{A}\left(e^{u}\right)$ has a jump. Hence we see that $s(u)$ belongs to the class $\left|T_{0}\right|$ where $\mu=1, \delta$ depends only on $c$. Since $\left|A_{\alpha}\right|$ summability implies $\left(A_{\alpha}\right)$ summability and the gap Tauberian theorem is true for $\left(A_{\alpha}\right)$ summability (vide [3]), $\sum a_{n}$ is convergent and hence follows the boundedness of the terms of $\sum a_{n}$. In view of (G), (4) now follows. (2) and (4) show that the integral (13) is absolutely convergent. All the requirements of Lemma 1 are satisfied and hence by the conclusion of the same lemma, we obtain

$$
\sum_{n=0}^{\infty}\left|a_{n}\right| \leqslant C \int_{-\infty}^{\infty}|g(v)| d v .
$$

Now

$$
\int_{-\infty}^{\infty}|g(v)| d v \leqslant \int_{-\infty}^{\infty}\left|g(v)-g_{1}(v)\right| d v+\int_{-\infty}^{\infty}\left|g_{1}(v)\right| d v,
$$


where $g_{1}(v)$ is given by (7). Then, substituting for $g(v)$ and $g_{1}(v)$ and applying Lemma 2, we get

$$
\begin{aligned}
& \int_{-\infty}^{\infty}|g(v)| d v \leqslant \int_{-\infty}^{\infty} d v \int_{-\infty}^{\infty}\left|k_{1}(v, u)-k(v-u)\right||d s(u)|+\int_{0}^{1}\left|f^{\prime}(x)\right| d x \\
& \quad \leqslant \int_{-\infty}^{\infty}|d s(u)| \int_{-\infty}^{\infty}\left|k_{1}(v, u)-k(v-u)\right| d v+\int_{0}^{1}\left|f^{\prime}(x)\right| d x \\
& \quad \leqslant \sum_{n=-\infty}^{\infty} \int_{n}^{n+1}|d s(u)| \max _{n \leqslant u<n+1} \int_{-\infty}^{\infty}\left|k_{1}(v, u)-k(v-u)\right| d v+\int_{0}^{1}\left|f^{\prime}(x)\right| d x \\
& \quad<M \sum_{n=-\infty}^{\infty} \max _{n \leqslant u<n+1} \int_{-\infty}^{\infty}\left|k_{1}(v, u)-k(v-u)\right| d v+\int_{0}^{1}\left|f^{\prime}(x)\right| d x \\
& \quad=M L+\int_{0}^{1}\left|f^{\prime}(x)\right| d x \quad \text { (by Lemma 3) }(4) \text { is satisfied) } \\
& \text { (15) }=C^{\prime}+\int_{0}^{1}\left|f^{\prime}(x)\right| d x .
\end{aligned}
$$

From (14) and (15), we obtain

$$
\sum_{n=0}^{\infty}\left|a_{n}\right|<C\left[C^{\prime}+\int_{0}^{1}\left|f^{\prime}(x)\right| d x\right]=C_{1}+C \int_{0}^{1}\left|f^{\prime}(x)\right| d x<\infty .
$$

The theorem is proved.

AcKnowledgement. I thank Dr. M. S. Rangachari and Professor V. K. Krishnan for their help in the preparation of this note, and the referee for simplifying the proof of Lemma 3.

\section{REFERENCES}

1. D. Borewein and J. H. Rizvi, On Abel type methods of summability, J. Reine Angew. Math. 247 (1971), 139-147.

2. G. H. Hardy, Divergent series, Oxford, England, 1949.

3. V. K. Krishnan, Gap Tauberian theorem for generalised Ahel summabilit, Math. Proc. Cambridge Philos. Soc. 78 (1975), 497-500.

4. V. I. Mel'nik, Tauberian theorem in the metric of L for convolution transforms, Ukrain. Mat. Z̆. 32 (1980), 831-836.

5. A. Zygmund, On certain integrals, Trans. Amer. Math. Soc. 55 (1944), 170-204.

Ramanujan Institute, University OF MADRAS, MADRAS-600 005, India 